\title{
Quick Delivery of Aldehyde Dehydrogenase into Yeast Vacuoles by QRPL Peptide Sequence on Carboxypeptidase $Y$
}

\section{Dong Jun Park}

Chonbuk National University

\section{Ngoc-Tu Nguyen}

Chonbuk National University

Ji Hun Kim

Korea Advanced Institute of Science and Technology

Ngoc-Han Nguyen

Chonbuk National University

\section{Sunchang Kim}

Korea Advanced Institute of Science and Technology

\section{Byung-Kwan Cho}

Korea Advanced Institute of Science and Technology

\section{Yang-Hoon Kim}

Chungbuk National University

Jiho Min ( $\sim$ jihomin@jbnu.ac.kr)

Chonbuk National University https://orcid.org/0000-0001-6025-7746

\section{Research article}

Keywords: Vacuolar targeting, Signal peptide sequence, Transfer vector, Target-delivery system, Carboxypeptidase Y, Aldehyde dehydrogenase 6 (ALD6)

Posted Date: July 9th, 2020

DOI: https://doi.org/10.21203/rs.3.rs-40710/v1

License: (c) (i) This work is licensed under a Creative Commons Attribution 4.0 International License. Read Full License 


\section{Abstract}

Background The signal peptide sequence is known to increase transport efficiency to organelles in eukaryotic cells. In this study, we focus on the signal peptide of the vacuolar protein for vacuolar targeting. Results The signal peptide sequence QRPL of carboxypeptidase Y (CPY), a vacuolar protein, was inserted inside the green fluorescent protein (GFP) that does not locate in vacuole for vacuolar targeting. The protein location was then confirmed by confocal microscopy. Fascinatingly, the green fluorescent protein that contains QRPL inside the sequence could be expressed faster than its natural form (within 1 hour after induction). In addition, aldehyde dehydrogenase 6 (ALD6), a cytosolic protein has engineered the sequence with QRPL to be transported to the vacuole. The aldehyde removal activity of ALD6 protein in the recombinant yeast was then analyzed by measuring the luminescent intensity in Vibrio fischeri . Conclusions In summary, the signal peptide QRPL could be used not only to transport target proteins accurately to vacuole but also to improve the protein activity, as well as to shorten the induction time.

\section{Background}

The vacuole is known as a cellular organelle in Saccharomyces cerevisiae. Many researchers have reported that vacuoles participate in vesicular trafficking processes ${ }^{1,2}$. The vacuole of $S$. cerevisiae contains about (50-60) acid hydrolases that play essential roles in several biological processes. Some special vacuolar enzymes can secrete cytotoxic molecules outside the cells. The degradable function of molecules typically takes place by endocytosis, after being activated as a response to stressful conditions ${ }^{3,4}$. In previous studies, we have investigated the antimicrobial activity of vacuoles from eukaryotes ${ }^{5,6}$. Here, we focus on the trafficking of vacuolar proteins by signal peptide sequence. Various signal peptide sequences that have been reported in most vacuolar enzymes are related to trafficking in cellular mechanisms ${ }^{7-10}$. Most vacuolar enzymes are delivered from the endoplasmic reticulum and trafficked to the Golgi complex, the pre-vacuole, or the late endosome. They are involved in the CPY pathway, in which proteins are transported from the Golgi complex to the vacuole ${ }^{9}$. Other known mechanisms of protein trafficking include the autophagy pathway, in which unnecessary vacuole or proteins can be converted into double-membraned autophagosomes ${ }^{10}$. In addition, the late endosome/multivehicle body pathway (MVB pathway) is related to delivery protein to the vacuole by endosomes. In a similar fashion, all proteins are transported in the cytosol by signaling mechanism. In 1980 , signal peptide sequences were intensively studied ${ }^{11-13}$. Specifically, vacuolar proteins contain various signal peptide sequences for protein trafficking in $S$. cerevisiae. Signal peptide sequences related to the transportation of protein or cargos to the vacuole and sorting mechanisms have been reported 12,13. In our study, we focus on the QRPL signal peptide sequence of Carboxypeptidase $Y$ (CPY), which is a typical vacuolar protein in $S$. cerevisiae ${ }^{14-18}$. Transfer vectors were designed by using QRPL to deliver the protein of interest to the vacuole that is not expressed in vacuole. QRPL has inserted in the middle of the CPY sequence at the 24 th to $27^{\text {th }}$ residues for the development of transfer vector. 
Furthermore, we tried to transport aldehyde dehydrogenase 6 (ALD6), which has been reported as an aldehyde removal protein through NAD-dependent reaction in the cytosol, to the vacuole ${ }^{19-22}$. Aldehydes are known to cause problems to the environment and animals including humans, by creating exogenous and endogenous precursors during numerous physiological processes ${ }^{22}$.

In this study, MBTL-Q-DJ1 and MBTL-Q-DJ2 were developed using the QRPL signal peptide sequence with target proteins as transfer vectors. Those protein expression levels were evaluated by fluorescence microscopy. MBTL-Q-DJ1 is a model of the transfer vector for the transport of GFP to the vacuole, and MBTL-Q-DJ2 contained ALD6 for the transport of a foreign protein to the vacuole by signal peptide sequence. Furthermore, the aldehyde removal efficiency of ALD6 was then evaluated. Therefore, this development of the transfer vector using a signal peptide sequence is useful for delivering proteins or peptide to the vacuole. In addition, protein that contains QRPL sequence could be expressed in vacuole after only 1 hour of induction. This investigation might be a potential approach for the therapy of vacuolar diseases.

\section{Results}

\section{Construction of pYES2::QRPL::GFP and pYES2::QRPL::ALD6::GFP for vacuolar targeting}

Carboxypeptidase Y (CPY) is known to be transported from ER to vacuole, including a signal peptide sequence near the N-terminal of the DNA fragment (QRPL sequence). In this study, we considered the possibility of the signal peptide delivering foreign proteins to the vacuole, after generation by the nucleus.

Here, the yeast vacuole was stained with LysoTracker-Red; the fluorescent signals of vacuole and GFP were observed by fluorescence microscopy, to confirm the location of GFP in the vacuole. The merged images show a yellow color indicating the combination of green and red color, whereas the mock strain did not show any green or yellow color. The result of the co-localization in vacuole means that GFP is successfully expressed in the vacuole (Fig. 2). Figures 2 (a) - (c) show the MBTL-M-DJ, while Figs. 2 (d) (f) show the MBTL-Q-DJ1. The fluorescence images of (a) and (d) show the vacuole by Lyso-Tracker, while (b) and (e) show the proteins of interest with GFP. Lastly, (c) and (f) show the co-localized images of vacuole and GFP.

In the same way, we inserted the QRPL sequence inside aldehyde dehydrogenase 6 (ALD6), followed by GFP to determine the localization of ALD6. Figures 2 (g) - (i) show the expression of ALD6 and GFP in the vacuole. The fluorescence images of (a) and ( $\mathrm{g}$ ) present the vacuole by Lyso-Tracker, while (b) and (h) show the proteins of interest with GFP. Lastly, (c) and (i) show the co-localized images of vacuole and GFP. There is no doubt that the signal peptide led the foreign proteins to the vacuole.

\section{Effect of QRPL sequence on the induction time for protein expression}

The induction with galactose is essential for protein expression using GAL promoter in pYES2 vector. A regular induction of GAL promoter in $S$. cerevisiae that was used in this study requires $(10-12) \mathrm{h}{ }^{23}$; thus 
we wanted to confirm whether the QRPL could provoke any effect on the induction process. Figure 3 shows different expression levels of green fluorescent protein after various induction times of $(0.5,1,3,6$, and 12) $\mathrm{h}$ of the strains MBTL-Q-DJ1 and MBTL-Q-DJ2. Interestingly, the yellow color in the merged images of recombinant yeasts provided that the GFP of MBTL-Q-DJ1 and ALD6 protein of MBTL-Q-DJ2 were successfully transported and expressed in the vacuole after $1 \mathrm{~h}$ of induction. These strains occupy QRPL signal peptide sequence in the middle of the DNA sequence that leads the proteins to the vacuole after only $1 \mathrm{~h}$, whereas the mock strain, which does not carry QRPL, could not show the co-localization of vacuole with the proteins of interest during induction.

\section{Aldehyde-reducing ability of the strain MBTL-Q-DJ2}

To evaluate the aldehyde dehydrogenase activity of ALD6 in the recombinant yeast, formaldehyde was exposed with the vacuolar enzymes that included ALD6 for $1 \mathrm{~h}$ at $62.5,125,250,500$, and 1,000 ppm. The mixture was then centrifuged at $20,000 \times g$ at $4^{\circ} \mathrm{C}$ for $10 \mathrm{~min}$ to remove pellet, and the supernatant collected for luminescent reaction with Vibrio fischeri. $V$. fischeri is known as having luminescence that could be decreased by being stressed. In this study, the remnant of aldehyde would react with the cell to reduce the luminescence. Table 1 shows the formaldehyde removal efficiency of MBTL-Q-DJ2 strain compared with the mock and control sample (no treatment), by measuring the luminescent intensity in $V$. fischeri. A high value of luminescence means $V$. fischeri was alive by reducing formaldehyde. The vacuolar enzyme that included ALD6 showed a high efficiency of decrease in formaldehyde at 62.5 and 125 ppm, whereas the cytoplasmic enzyme did not show any influence.

Table 1

The formaldehyde removal ability of ALD6. The value in the table presents the luminescent intensity of all samples; "control" means sample without treatment. The decreased efficiency of aldehyde was compared with those of mock and recombinant strain by measuring the luminescent intensity in $V$. fischeri. The high value of luminescence means $V$. fischeri was alive by reducing formaldehyde. ALD 6 showed excellent efficiency to decrease formaldehyde at ( 62.5

and 125) ppm, while the cytoplasmic enzyme did not show any reduction of formaldehyde effect.

\begin{tabular}{|llllll|}
\hline & & \multicolumn{2}{c}{ Vacuolar enzyme } & \multicolumn{2}{c|}{ Cytoplasmic enzyme } \\
\hline Formaldehyde (ppm) & Control & Mock & Expressed ALD6 & Mock & Expressed ALD6 \\
\hline 0 & 1 & 1 & 1 & 1 & 1 \\
\hline 62.5 & 0.1114 & 0.8804 & 0.9867 & 0.9135 & 0.9004 \\
\hline 125 & 0.0546 & 0.5487 & 0.6077 & 0.5670 & 0.5451 \\
250 & 0.0018 & 0.1892 & 0.1733 & 0.1793 & 0.1754 \\
\hline 500 & 0.0005 & 0.0613 & 0.0662 & 0.0519 & 0.0398 \\
\hline
\end{tabular}

Furthermore, it is necessary to determine the optimized concentration of vacuolar enzymes for the highest reduction effect. Figure 4 presented the luminescent level after exposing formaldehyde to 
different concentrations of vacuolar enzymes of 300,500 and $1,000 \mu \mathrm{g} / \mu \mathrm{L}$. The highest gap of luminescence was observed at $500 \mu \mathrm{g} / \mu \mathrm{L}$ of the vacuolar enzyme from MBTL-Q-DJ2.

\section{Discussion}

Many proteins contain signal peptide sequences, which enable them to be delivered accurately for cellular mechanisms. The delivery of proteins from the late Golgi in yeast depends on which protein trafficking pathway that targets protein is involved. In this work, the signal peptide sequence of vacuolar protein Carboxypeptidase Y (CPY) of S. cerevisiae, QRPL, was used. Even though QRPL was investigated a long time ago, this sequence was not studied in the protein targeting field. Here, the transfer vector that was designed with QRPL signal peptide sequence demonstrated the capability of leading GFP and ALD6, a cytosolic protein, to the vacuole. Therefore, this transfer vector could be used to deliver the protein of interest or peptide to the yeast vacuole.

Interestingly, QRPL was found to accelerate the induction process of the protein of interest in the vacuole. The recombinant yeast $S$. cerevisiae $\mathbf{2} 2805$ that contains the plasmid pYES2 with GAL promoter regularly requires $(10-12)$ h of galactose induction for the protein expression ${ }^{23}$; however, the presence of QRPL in the recombinant yeast quickened the protein expression, which was observed $1 \mathrm{~h}$ after adding galactose. Further investigation with a shorter induction time should be considered for clearer understanding of this phenomenon.

In conclusion, we designed a useful transfer vector that could deliver a target protein to the vacuole using the signal peptide sequence of the vacuolar protein. The signal peptide sequence of CPY, QRPL was inserted at the 24th to 27th residues with the target protein, and it was possible to transport it to the vacuole. Therefore, our investigation provided a promising approach for protein targeting that could be applied in varied fields.

\section{Materials And Methods}

\section{Culture of S. cerevisiae, chemicals, and materials}

Saccharomyces cerevisiae 2805 was kindly purchased and provided by the Korea Research Institute of Bioscience and Biotechnology (KRIBB) in Korea. S. cerevisiae was transformed with pYES2.0 plasmid for mock strain, and pYES2::QRPL:::GFP, pYES2::QRPL::ALD6::GFP plasmid for recombinant strain. Mock and recombinant strains were cultured in SD medium ( $0.67 \%$ yeast nitrogen base, $0.5 \%$ casamino acid, and 2 $\%$ D-glucose). To induce the activation of GAL promoter on pYES2.0 plasmid, the medium was changed to SG medium ( $0.67 \%$ yeast nitrogen base, $0.5 \%$ casamino acid, and $2 \% \mathrm{D}$-galactose) for $12 \mathrm{~h}$. Formaldehyde $(1.09 \mathrm{~g} / \mathrm{mL})$ was purchased from Sigma Chemical Co. (St. Louis, USA).

\section{DNA isolation, manipulation, and transformation}


Genomic DNA was prepared from S. cerevisiae s2805 using Wizard SV genomic DNA kit (Promega, Madison, USA), according to the manufacturer's instructions. Plasmid DNA, such as pYES2.0 (Invitrogen, USA) and pEGFP-C1 (Clontech Laboratories, Inc., USA), was prepared from recombinant E. coli cells using an alkaline lysis technique with a QIA spin Miniprep kit (Qiagen, Germany). All of the DNA modifications were analyzed by agarose gel electrophoresis, and ligation was performed using standard procedures (Sambrook et al., 2001). The PCR experiments were carried out using a T Gradient Thermocycler (Biometra, Germany), Ex TaqTM DNA polymerase (Takara Bio Inc., Japan), and genomic DNA as the template.

The PCR products were purified using a QIA quick PCR purification kit (Qiagen, Germany). The oligonucleotides used for PCR amplification were purchased from AccuOligo (Bioneer Co., Korea). Figure 1 describes two diagrams of the transfer vector with QRPL signal peptide sequence (QRPL). The transformation to $E$. coli was performed by electroporation with an Electro cell Manipulator (BTX Technologies Inc., USA); and yeast transformation was performed by lithium acetate method ${ }^{24}$.

\section{Design of transfer vector (pYES2::QRPL::GFP)}

Plasmids used in this study were constructed for the expression of QRPL signal peptide sequence with green fluorescence protein (GFP) (Fig. 1 a). To amplify the QRPL gene (5'- CAA CGG TCT TTG - 3'), the primer was designed to include QRPL signal peptide sequence from CPY in the PYES2 plasmid. PCR products were prepared of the two parts of the gene, then primer R1 and F2 included QRPL with Earl restriction enzyme site. PCR via primer: Primer-F1 (5'- TTT AGC GGC CGC ATG GTG AGC AAG GGC GAG $-3^{\prime}$ ) and Primer-R1 (5'- ATA CTC TTC CAA CGG TCT TTG TAC GTC GCC GTC CAG CTC - $3^{\prime}$ ). The PCR product was prepared with restriction enzyme site Notl and Earl sites. In addition, Primer-F2 (5'- TTT CTC TTC CAA AGA CCG TTG AAC GGC CAC AAG TTC AGC -3') and Primer-R2 (5'- ATT GCA TGC TTA CTT GTA CAG CTC GTC -3') were designed with restriction enzyme site Earl sites and Sphl. Underline on the primer R1 and F2 indicates QRPL sequence. The fusion PCR fragment was then cloned into the Notl and Sphl site to make pYES2::QRPL::GFP. This vector was transformed into $S$. cerevisiae cells to make recombinant strain MBTL-Q-DJ1. In parallel, the original vector pYES2.0 was transformed into yeast to make mock strain MBTL-M-DJ.

\section{Design of transfer vector (pYES2::QRPL::ALD6::GFP)}

Plasmids used in this study were constructed for the expression of QRPL signal peptide sequence with Aldehyde dehydrogenase 6 (ALD6). The DNA containing QRPL with ALD6 was synthesized by Bioneer Co., Korea. The PCR product was inserted into the BamHI and Not/ site of pYES2.0, to make pYES2::QRPL::ALD6 plasmid. After that, the GFP gene was amplified from pEGFP using PCR via primers: Primer-GFP-F (5'- ATT GCG GCC GCA GTG AGC AAG GGC GAG G -3') and Primer-GFP-R (5'- GGT GCA TGC CTT GTA CAG CTC ATC CAT -3'). The DNA fragment of GFP was then cloned into the Not/ and Sph/site to make pYES2::QRPL::ALD6::GFP. This vector was transformed into $S$. cerevisiae to make recombinant strain MBTL-Q-DJ2 (Fig. 1 b). 


\section{Fluorescence microscopy}

Recombinant and mock strains were cultured in SD and SG medium. The cells were washed with PBS buffer and stained with 100 nM Lyso-Tracker Red DND-99 (Molecular Probes, Leiden, The Netherlands) in PBS for $30^{\circ} \mathrm{C}$ for $10 \mathrm{~min}$. All strains were washed by PBS twice, and the intensity of protein expression observed by fluorescence microscopy (Zeiss, Germany). Fluorescent images were analyzed using the Zeiss image Browser.

\section{Isolation of vacuole and vacuolar enzyme from recombinant yeast}

Recombinant and mock strains were both cultured in SD medium. To induce pYES2.0 plasmid, cells were cultured on SG medium for $12 \mathrm{~h}$. All strains were harvested after growing at $\mathrm{OD}_{600} 0.8-1.0$, then the vacuole was isolated from mock and recombinant strains.

The $0.1 \mathrm{M}^{\text {Tris-SO }} \mathrm{S}_{4}$ buffer $\left(100 \mathrm{mM}\right.$ Tris-SO ${ }_{4}(\mathrm{pH} 9.4), 10 \mathrm{mM}$ dithiothreitol (DTT)) was mixed with harvesting cells. This mixture was incubated at $30^{\circ} \mathrm{C}$ at $90 \mathrm{rpm}$ for $10 \mathrm{~min}$ and centrifuged at 3,000 rpm for $5 \mathrm{~min}$. The supernatant was discarded, and the mixed pellet exposed to Lyticase $(2,000 \mathrm{U} / \mathrm{mL})($ Sigma, St. Louis, USA) for $1 \mathrm{~h}$, to make it flexible in cell membranes. This was followed by centrifugation for 5 min, and the pellet was washed twice with Sorbitol $\mathrm{K}^{+}$phosphate buffer $\left(20 \mathrm{mM} \mathrm{K}^{+}\right.$phosphate buffer, 1.2 M Sorbitol). In the next step, breaking buffer (20 mM Tris- $\mathrm{Cl}$ (pH 7.4), $0.6 \mathrm{mM}$ Sorbitol, and $1 \mathrm{mM}$ phenyl methane sulfonyl fluoride (PMSF)) was mixed with pellet, and ultra-sonication applied at $40 \mathrm{~W}$ for 30 min (20 s on / $10 \mathrm{~s}$ off pulse) in the ice. The mixture was centrifuged for $10 \mathrm{~min}$, and the supernatant was discarded. The pellet was mixed again with breaking buffer, and a second ultra-sonication was applied for $20 \mathrm{~min}$ ( $10 \mathrm{~s}$ on / $10 \mathrm{~s}$ off pulse). Lastly, the first centrifuge ( $500 \times \mathrm{g}, 5 \mathrm{~min}$ ) was made to collect supernatant, and the second centrifuge $\left(20,000 \times \mathrm{g}\right.$, was made for $30 \mathrm{~min}$ at $\left.4{ }^{\circ} \mathrm{C}\right)$. The pellet contains vacuoles from cells $5,25,26$.

The vacuolar enzyme was extracted by $0.1 \%$ NP40 buffer containing 1 mM DTT at a ratio of 1:1. The mixture was vortexed for $10 \mathrm{~min}$, followed by the reaction in ice for $30 \mathrm{~min}$. After incubation, the mixture was centrifuged at $13,000 \mathrm{rpm}$ at $4{ }^{\circ} \mathrm{C}$ for $10 \mathrm{~min}$. The supernatant contained vacuolar enzymes that would be used in the aldehyde reduction assay.

\section{Luminescence assay}

The vacuolar enzyme was exposed to formaldehyde to evaluate the efficiency of aldehyde reduction. Formaldehyde was prepared at $62.5,125,250,500$, and $1,000 \mathrm{ppm}$, and then the vacuolar enzyme was exposed to the formaldehyde at room temperature for $1 \mathrm{~h}$. After that, the mixture was centrifuged at $20,000 \times \mathrm{g}$ at $4{ }^{\circ} \mathrm{C}$ for $10 \mathrm{~min}$, the supernatant was collected for luminescent reaction with Vibrio fischeri at a ratio of $1: 1$ at $30^{\circ} \mathrm{C}$ for $30 \mathrm{~min}$, and the luminescence was measured by GloMax ${ }^{\circledR}$ Explorer system (Promega Co., Ltd., USA) ${ }^{27,28 .}$

\section{Data analysis}


All the data were obtained from three independent samples, run simultaneously for error analysis. The data were analyzed using Sigma Plot (SPS, Chicago, IL. USA), and the results were reported with the standard deviations. In addition, the correlation between cell viability and several experimental conditions was reported. A $p$-value of $<0.05$ was considered significant.

\section{Abbreviations}

ALD6: aldehyde dehydrogenase 6

CPY: carboxypeptidase $Y$

GFP: green fluorescent protein

\section{Declarations}

\section{Acknowledgment}

Not applicable.

\section{Author contributions}

D.J.P and J.M designed the research project. D.J.P conducted the experiments and analyzed the data. D.J.P and N.T.N prepared the manuscript. Y.H.K and J.M finalized the manuscript. All authors read and approved the final manuscript.

\section{Funding}

This research was supported by the Basic Science Research Program through the National Research Foundation of Korea (NRF), funded by the Ministry of Science, ICT \& Future Planning (2017R1A2B4009775).

\section{Availability of data and materials}

The datasets used and analyzed during the current study are available from the corresponding author on reasonable request.

\section{Competing interests}

The authors declare that they have no competing interests.

\section{Ethics approval and consent to participate}

Not applicable.

Consent for publication 
Not applicable.

\section{References}

1. Armstrong J. Yeast vacuoles: more than a model lysosome. Trends Cell Biol. 2010;20(10):580-5.

2. Wickner W. Yeast vacuoles and membrane fusion pathways. The EMBO Journal. 2002;21(6):1241-7.

3. Li SC, Kane PM. The yeast lysosome-like vacuole: endpoint and crossroads. BBA-Mol Cell Res. 2009;1793(4):650-63.

4. Tardy C, Codogno P, Autefage H, Levade T, Andrieu-Abadie N. Lysosomes and lysosomal proteins in cancer cell death (new players of an old struggle). BBA-Rev Cancer. 2006;1765(2):101-25.

5. Yoon J, Park J-M, Jung S-K, Kim K-Y, Kim Y-H, Min J. Characterization of antimicrobial activity of the lysosomes isolated from Saccharomyces cerevisiae. Curr Microbiol. 2009;59(1):48-52.

6. Yoon J, Chang S-T, Park J-S, Kim Y-H, Min J. Functional characterization of starvation-induced lysosomal activity in Saccharomyces cerevisiae. Appl Microbiol Biotechnol. 2010;88(1):283-9.

7. Saftig P, Klumperman J. Lysosome biogenesis and lysosomal membrane proteins: trafficking meets function. Nat Rev Mol Cell Bio. 2009;10(9):623.

8. Boya P. Lysosomal function and dysfunction: mechanism and disease. Antioxid Redox Sign. 2012;17(5):766-74.

9. Rothman JH, Howald I, Stevens T. Characterization of genes required for protein sorting and vacuolar function in the yeast Saccharomyces cerevisiae. The EMBO journal. 1989;8(7):2057-65.

10. Bowers K, Stevens TH. Protein transport from the late Golgi to the vacuole in the yeast Saccharomyces cerevisiae. BBA-Mol Cell Res. 2005;1744(3):438-54.

11. Ni X, Canuel M, Morales CR. The sorting and trafficking of lysosomal proteins. Histol Histopathol. 2006.

12. Conibear E, Stevens TH. Vacuolar biogenesis in yeast: sorting out the sorting proteins. Cell. 1995;83(4):513-6.

13. Wendland B, Emr SD, Riezman H. Protein traffic in the yeast endocytic and vacuolar protein sorting pathways. Curr Opin Cell Biol. 1998;10(4):513-22.

14. Saalbach G, Jung R, Kunze G, Saalbach I, Adler K, Müntz K. Different legumin protein domains act as vacuolar targeting signals. The Plant Cell. 1991;3(7):695-708.

15. Hong E, Davidson AR, Kaiser CA. A pathway for targeting soluble misfolded proteins to the yeast vacuole. J Cell Biol. 1996;135(3):623-33.

16. Valls LA, Winther JR, Stevens TH. Yeast carboxypeptidase Y vacuolar targeting signal is defined by four propeptide amino acids. J Cell Biol. 1990;111(2):361-8.

17. van Voorst F, Kielland-Brandt MC, Winther JR. Mutational analysis of the vacuolar sorting signal of procarboxypeptidase $Y$ in yeast shows a low requirement for sequence conservation. J Biol Chem. 1996;271(2):841-6. 
18. Klionsky D, Emr SD. A new class of lysosomal/vacuolar protein sorting signals. J Biol Chem. 1990;265(10):5349-52.

19. Singh S, Brocker C, Koppaka V, et al. Aldehyde dehydrogenases in cellular responses to oxidative/electrophilicstress. Free Radical Bio Med. 2013;56:89-101.

20. Grün F, Hirose Y, Kawauchi S, Ogura T, Umesono K. Aldehyde dehydrogenase 6, a cytosolic retinaldehyde dehydrogenase prominently expressed in sensory neuroepithelia during development. J Biol Chem. 2000;275(52):41210-8.

21. Marchitti SA, Brocker C, Stagos D, Vasiliou V. Non-P450 aldehyde oxidizing enzymes: the aldehyde dehydrogenase superfamily. Expert Opin Drug Met. 2008;4(6):697-720.

22. Nunoshiba T, Hashimoto M, Nishioka H. Cross-adaptive response in Escherichia coli caused by pretreatment with $\mathrm{H} 2 \mathrm{O} 2$ against formaldehyde and other aldehyde compounds. Mutat Res-DNA Repair. 1991;255(3):265-71.

23. Nguyen N-T, Park R-M, Kim Y-H, Min J. Detection and discrimination of Shigella sonnei and Shigella flexneri based on vacuolar responses in Saccharomyces cerevisiae. J Biotechnol. 2018;287:1-7.

24. Schiestl RH, Gietz RD. High efficiency transformation of intact yeast cells using single stranded nucleic acids as a carrier. Curr Genet. 1989;16(5-6):339-46.

25. Ferro-Novick S, Novick P, Field C, Schekman R. Yeast secretory mutants that block the formation of active cell surface enzymes. J Cell Biol. 1984;98(1):35-43.

26. Haas A, Scheglmann D, Lazar T, Gallwitz D, Wickner W. The GTPase Ypt7p of Saccharomyces cerevisiae is required on both partner vacuoles for the homotypic fusion step of vacuole inheritance. The EMBO journal. 1995;14(21):5258-70.

27. Bose JL, Kim U, Bartkowski W, et al. Bioluminescence in Vibrio fischeri is controlled by the redoxresponsive regulator ArcA. Mol Microbiol. 2007;65(2):538-53.

28. Lupp C, Urbanowski M, Greenberg EP, Ruby EG. The Vibrio fischeri quorum-sensing systems ain and lux sequentially induce luminescence gene expression and are important for persistence in the squid host. Mol Microbiol. 2003;50(1):319-31.

\section{Figures}


(a)

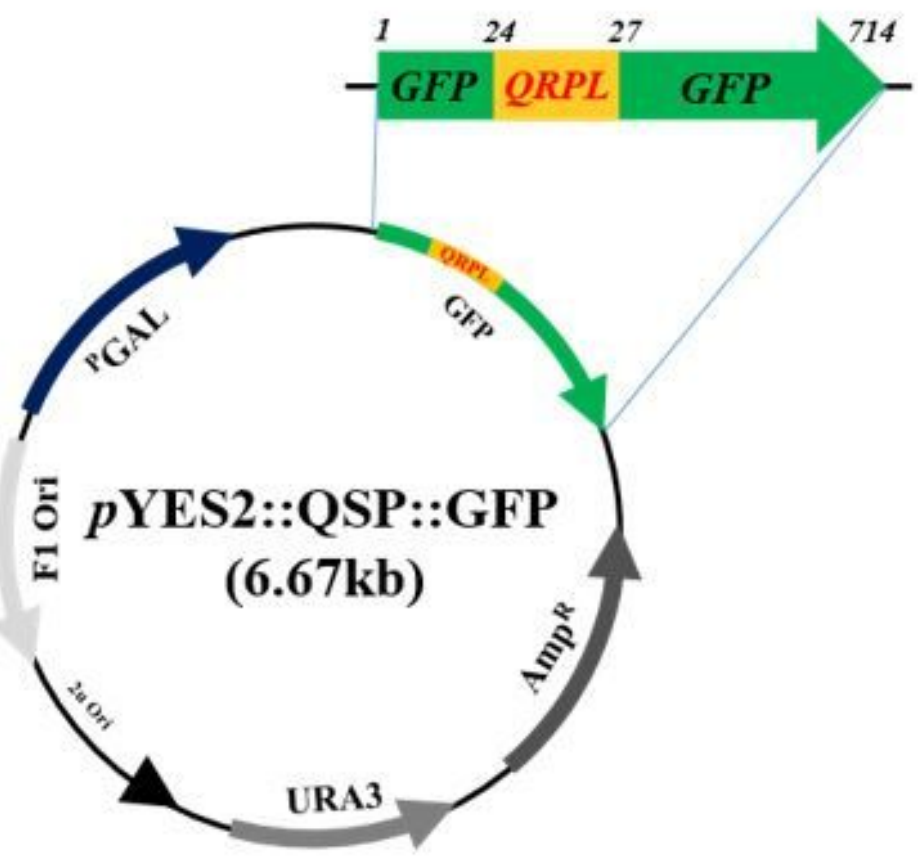

(b)

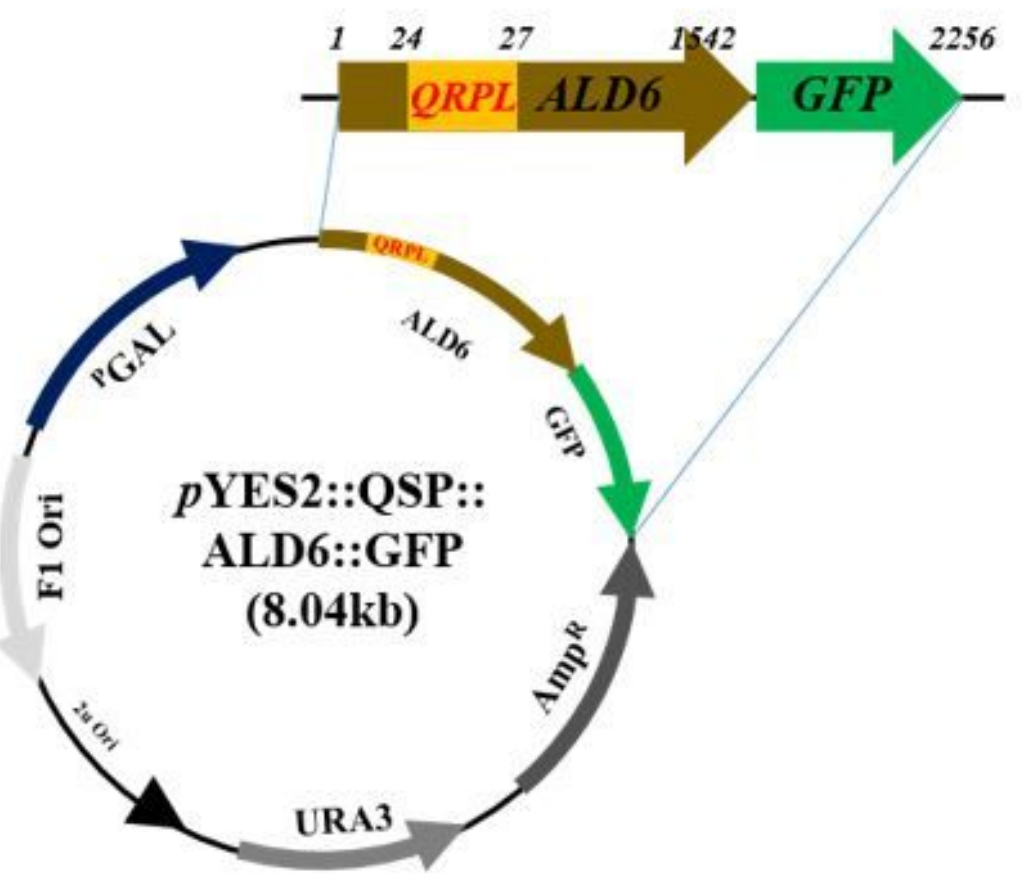

Figure 1

The designed recombinant plasmids that contain QRPL signal peptide sequence. (a) QRPL is inserted to become the 24th and 27th amino acids of GFP. This transfer vector is called MBTL-Q-DJ1. (b) QRPL is inserted to become the 24th and 27th amino acids of Aldehyde dehydrogenase 6 (ALD6). This transfer vector is called MBTL-Q-DJ2. 


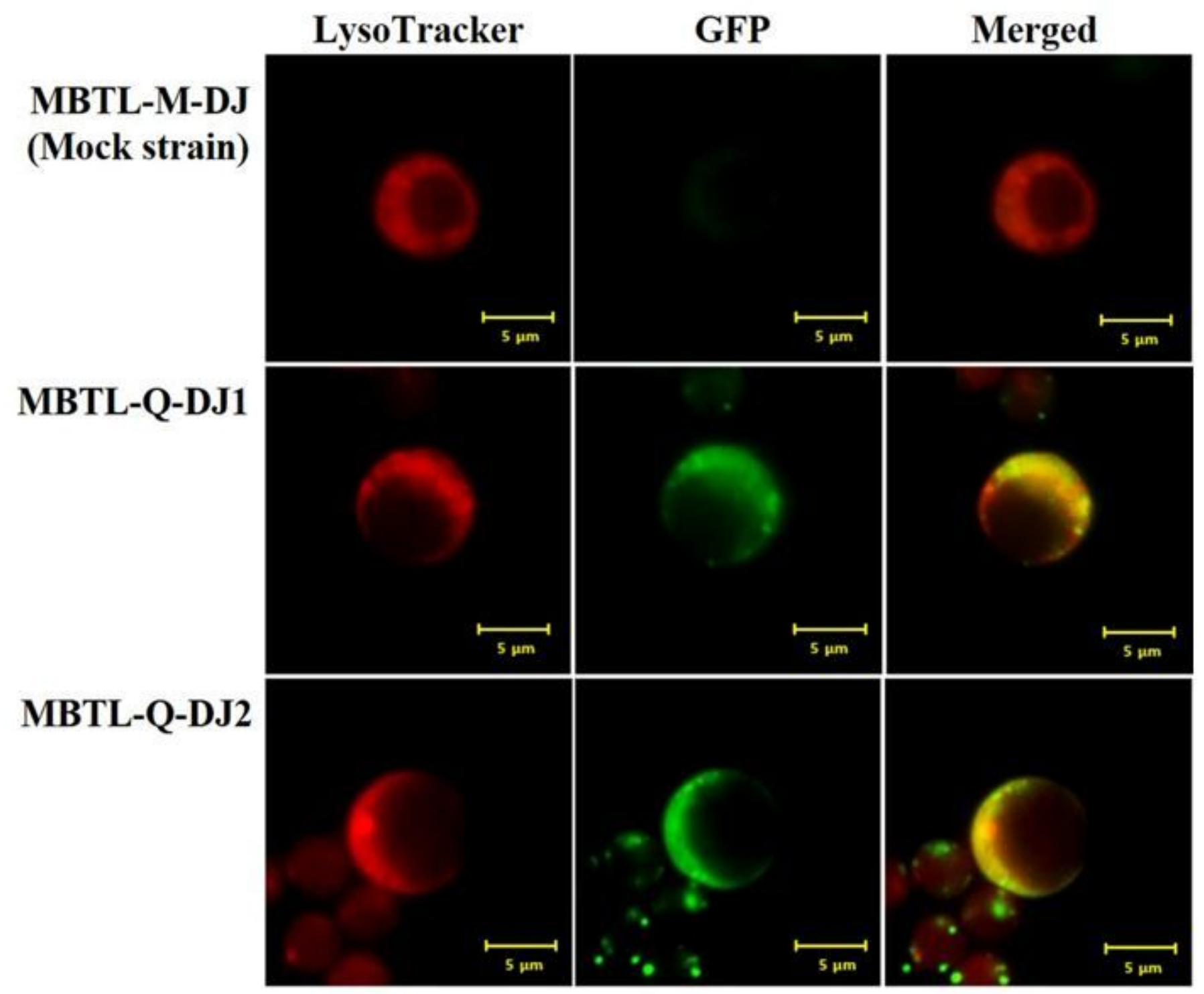

Figure 2

The expression of target proteins in vacuoles of the mock strain (control), MBTL-Q-DJ1 and MBTL-Q-DJ2. The "LysoTracker" column presents the red signal of yeast vacuoles after staining with LysoTracker-Red; the "GFP" column shows the green fluorescent signals, while the "Merged" column means the combination of red and green signals, which reflects the expression of GFP in vacuoles. 


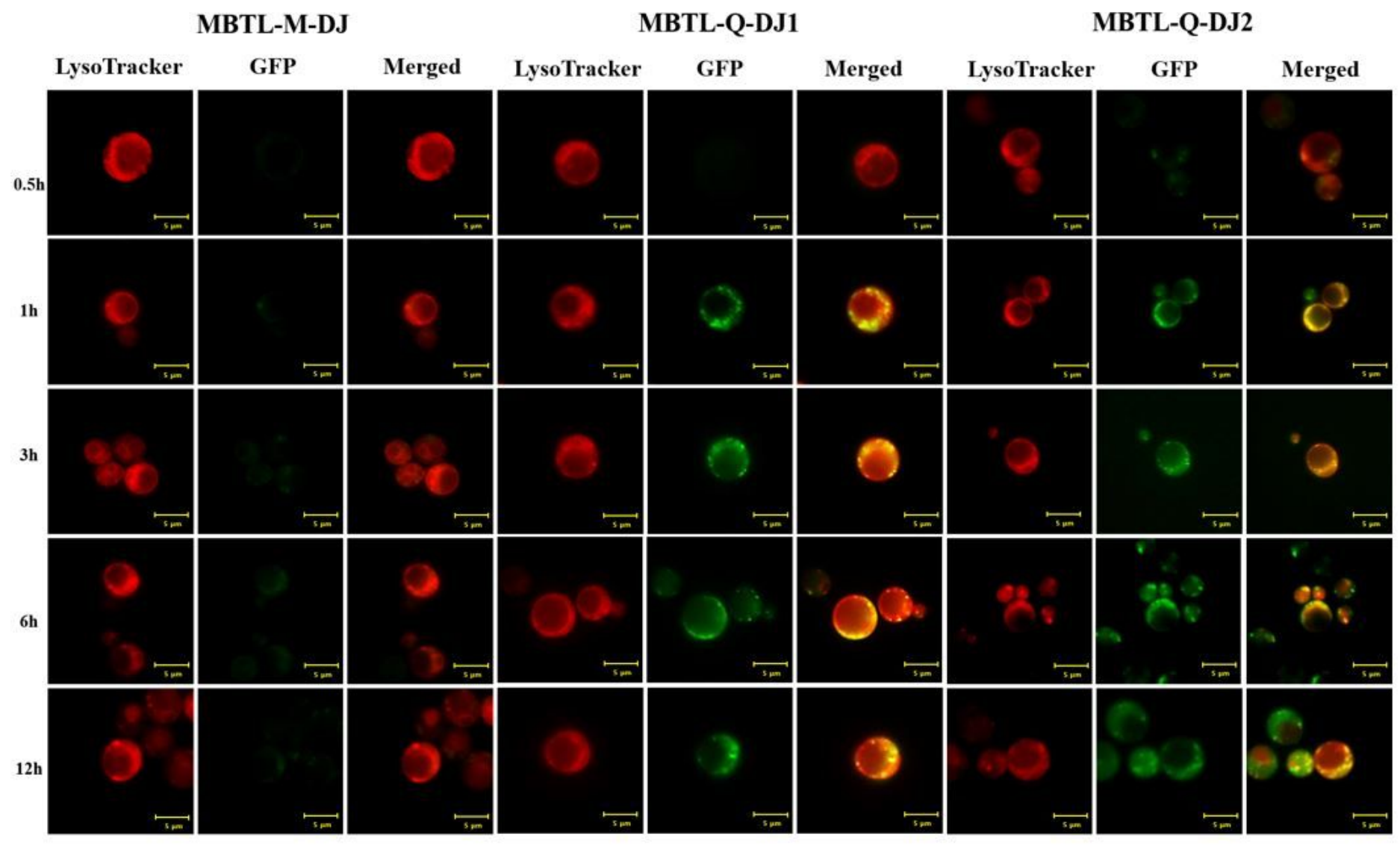

Figure 3

The protein expression at different induction time by galactose of MBTL-M-DJ, MBTL-Q-DJ1, and MBTLQ-DJ2. The "LysoTracker" column presents the red signal of yeast vacuoles after staining with LysoTracker-Red; the "GFP" column shows the green fluorescent signals, and the "Merged" column means the combination of red and green signals, which reflects the expression of GFP in vacuoles. 


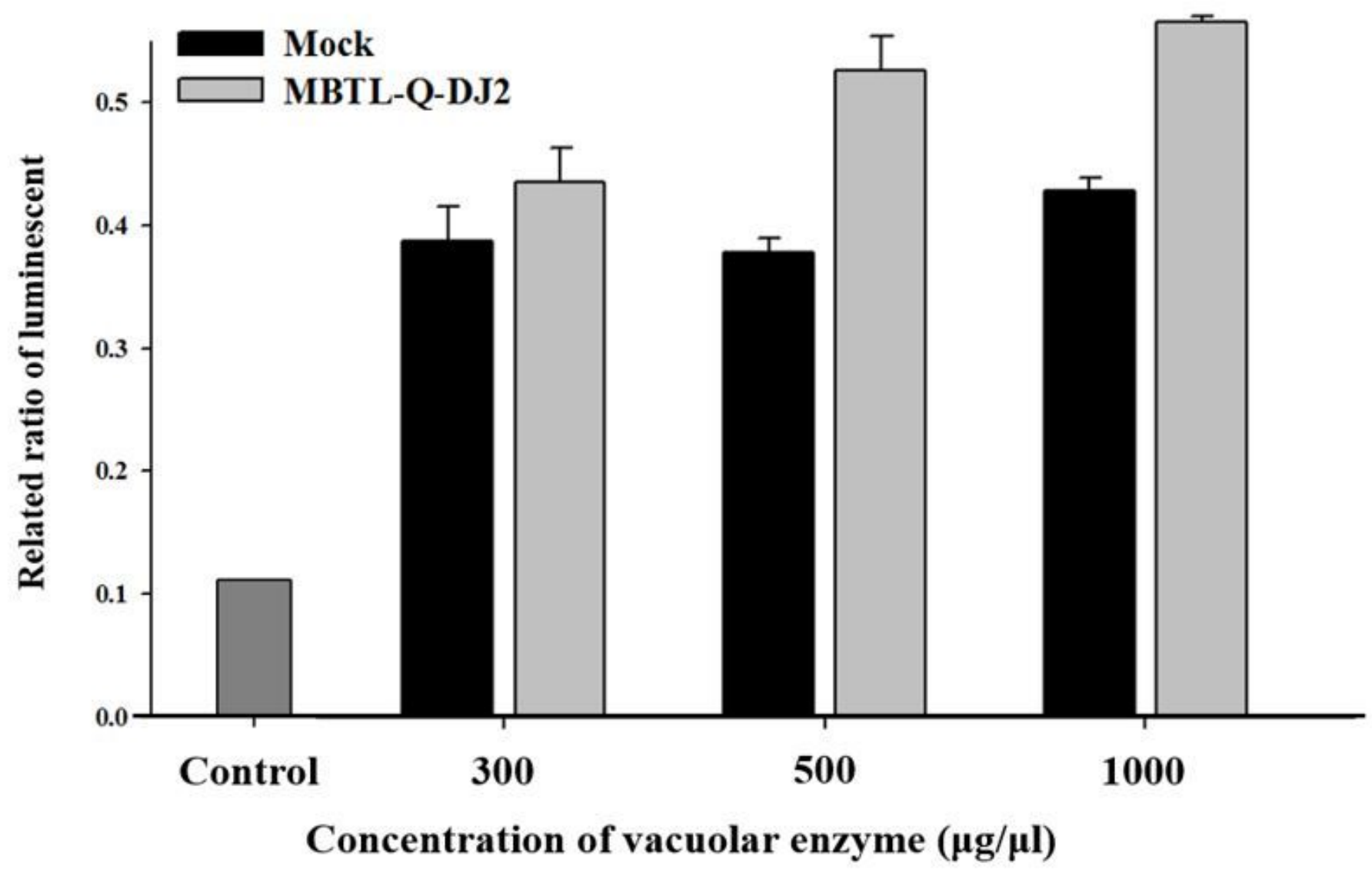

Figure 4

The aldehyde decreasing capacity by various concentrations of vacuolar enzymes isolated from MBTL-QDJ2. The data were analyzed using Sigma Plot (SPS Chicago, IL. USA), and a p-value $<0.05$ was considered significant. 